\title{
FACTORES DE RIESGO CARDIOVASCULAR EN RESIDENTES Y CARDIÓLOGOS ARGENTINOS. "CÓMO SE CUIDAN LOS QUE CUIDAN". ENCUESTA FRICCAR, CONAREC
}

\author{
CARDIOVASCULAR RISK FACTORS IN ARGENTINE RESIDENTS AND \\ CARDIOLOGISTS. "HOWTO CARE FOR THOSE WHO PROVIDE CARE". \\ FRICCAR SURVEY, CONAREC
}

HERNÁN PULENTA, LUCRECIA M. BURGOS, IGNACIO CIGALINI, DANIEL LORENZATTI, IVAN VILAR, MAURO ROSSI PRAT, FRANCO GIANNASI, ELISA ORTIZ, AGUSTÍN ROUDE, SEBASTIÁN GARCÍA-ZAMORA*

\section{RESUMEN}

Introducción. Si bien se ha avanzado en el conocimiento y tratamiento de los factores de riesgo cardiovascular (FRCV) en población general, se conoce menos respecto de la distribución de estos entre médicos.

Objetivos. Describir los FRCV presentes en residentes de cardiología y cardiólogos pertenecientes a residencias de Cardiología de todo el país.

Métodos. Se realizó una encuesta cerrada y prefijada, voluntaria y anónima, con un muestreo no probabilístico por conveniencia, en centros pertenecientes a Consejo Argentino de Residentes de Cardiología (CONAREC), durante los meses de agosto-noviembre de 2017.

Resultados. Se encuestaron 523 profesionales pertenecientes a 44 centros de todo el país. El 63,1\% de los participantes fueron hombres, y la mediana de edad fue 31 años (rango intercuartílico [RIC]: 28-38 años). El 52,8\% eran residentes, $5,9 \%$ jefes o instructores de residentes y $41,3 \%$ especialistas o fellow. Los princi pales FRCV detectados fueron: dislipemia 14,3\%, hipertensión arterial 5\%, enfermedad coronaria previa $1 \%$ y diabetes mellitus tipo $\| 0,8 \%$. Asimismo fumaban $16,8 \%$, y $12,6 \%$ eran extabaquistas. El $36,7 \%$ de los participantes manifestó no hacer ningún tipo de actividad física, y 33,8\% expresó que nunca se cuidaba de no agregar sal a los alimentos. Además, 35,6\% expresó que no comían frutas ni verduras diariamente. La mediana de estatura de las mujeres fue 1,63 metros (RIC 1,6-1,68), mientras que la de los hombres fue 1,75 metros (RIC: 1,71-1,81); la mediana del peso de las mujeres fue $58 \mathrm{~kg}$ (RIC: 55-63), en tanto la de los hom bres fue $81 \mathrm{~kg}$ (RIC: 73-90). La mediana del índice de masa corporal fue 21,7 (RIC: 20,3-24) para mujeres y 26,1 (RIC: $24,2-28,1$ ) en hombres. De acuerdo con esto, $46,5 \%$ de los encuestados cumplían criterio de sobrepeso u obesidad. El perímetro abdominal de los hombres fue $90 \mathrm{~cm}$ (RIC: 84-99) y el de las mujeres 70 (RIC 66-78). Respecto de los valores de presión arterial, el promedio sistólico fue de $115,7 \pm 11,9 \mathrm{mmHg}$ y diastólico $71,6 \pm 8,6 \mathrm{mmHg}$. Por otra parte, el 94,7\% de los participantes manifestó tener algún tipo de cobertura médica, al tiempo que $41,9 \%$ del total de encuestados reconoció que había pasado más de 1 año desde su último control de salud.

Conclusión. Encontramos una elevada tasa de sobrepeso y obesidad entre los participantes, como también de sedentarismo, tabaquismo y dislipemia. Si bien la prevalencia de otros factores de riesgo fue baja, debe tenerse presente que los participantes fueron en su mayoría jóvenes. Consideramos esencial implementa estrategias para favorecer la práctica de estilos de vida más favorables entre profesionales de la salud de nuestro país.

Palabras clave: factores de riesgo; obesidad, hipertensión, tabaquismo, hipercolesterolemia, cardiología.

\section{ABSTRACT}

Introduction: Although there has been progress in the knowledge and treatmen of cardiovascular risk factors (CVRF) in the general population, less is known about the distribution of these among physicians.

Objectives: To describe the CVRF present in residents of cardiology and cardiologists belonging to Cardiology residences throughout the country.

Methods: A closed and pre-determined, voluntary and anonymous survey was carried out with a non-probabilistic sampling for convenience in centers belonging to the Argentine Council of Cardiology Residents (CONAREC), during the months of August-November of 2017.

Results: 523 professionals belonging to 44 centers across the country were surveyed. $63.1 \%$ of the participants were men, and the median age was 31 years (interquartile range $[\mathrm{RIC}]$ 28-38 years). 52.8\% were residents, 5.9\% chiefs or instructors of residents and $41.3 \%$ specialists or fellows. The main CVRF detected were: dys lipidemia $14.3 \%$, hypertension $5 \%$, previous coronary disease $1 \%$ and Diabetes Mellitus type II $0.8 \% .16 .8 \%$ also smoked, and $12.6 \%$ were former smokers. $36.7 \%$ of the participants said they did not do any kind of physical activity, and $33.8 \%$ said they never took care not to add salt to food. In addition, $35.6 \%$ said they did not eat fruits or vegetables daily. The median height of the women was $1.63 \mathrm{me}$ ters (IQR 1.6-1.68), while that of the men was 1.75 meters (IOR 1.71-1.81): the median weight of women was $58 \mathrm{~kg}$ (IQR 55-63), while that of men was $81 \mathrm{~kg}$ (IQR 73-90). The median body mass index was 21.7 (IQR 20.3-24) for women and 26.1 (IQR 24.2-28.1) for men. According to this, $46.5 \%$ of the respondents met criteria of overweight or obesity. The abdominal perimeter of men was $90 \mathrm{~cm}$ (IQR 84-99) and that of women 70 (IQR 66-78). Regarding blood pressure values, the systolic average was $115.7 \pm 11.9 \mathrm{mmHg}$ and diastolic $71.6 \pm 8.6 \mathrm{mmHg}$. On the other hand, $94.7 \%$ of the participants said they had some type of medical coverage, while $41.9 \%$ of the total respondents admitted that more than 1 year had passed since their last health check-up.

Conclusion: We found a high rate of overweight and obesity among the participants, as well as sedentary lifestyle, smoking and dyslipidemia. Although the prevalence of other risk factors was low, it should be kept in mind that the participants were mostly young. We consider it essential to implement strategies to favor the practice of more favorable lifestyles among health professionals in ou country.

Keywords: risk factors; obesity; hypertension; tobacco use disorder; hypercholes terolemia; cardiology

REVISTA CONAREC 2018;33(147):308-312 | DOI:10.32407/RCON/2018147/0308-0312

\footnotetext{
* En representación de CONAREC

$\triangle$ Correspondencia:Dr. Hernán Pulenta. Consejo Argentino de Residentes de Cardiología, Azcuénaga 980, C1115AAD CABA, Rep.Argentina.pablohernanpulenta@gmail.com
}

La línea cardiovascular y metabólica de Laboratorio Roemmers participó de la ideación del proyecto, la confección del CRF y de la implementación del proyecto. Sin embargo, no participaron del procesamiento de datos ni de la redacción de resultados y conclusiones.

Recibido: 28/12/2018| Aceptado: 30/12/2018

\section{INTRODUCCIÓN}

La aterosclerosis es una compleja enfermedad multifactorial que involucra una interacción de factores genéticos y ambientales, y es la principal causa de morbimortalidad y discapacidad en la sociedad actual $^{1,2}$. Afecta a arterias de diferentes localizaciones simultáneamente pero con diferente grado de progresión $n^{3}$. Sus manifestaciones clí- 


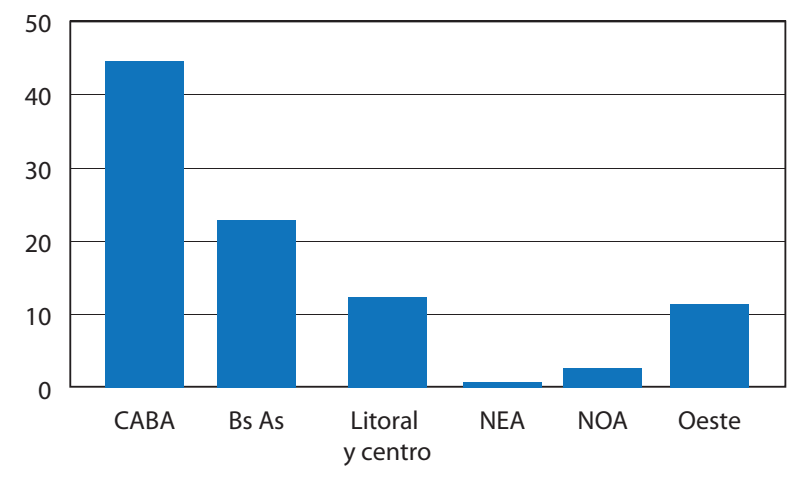

Figura 1. Distribución por regiones de los encuestados. Abreviaturas: CABA: Ciudad de Buenos Aires; Bs. As: Provincia de Buenos Aires y Gran Buenos Aires; NEA: Noreste Argentino; NOA: Noroeste Argentino. Provincias y ciudades incluidas según región: Litoral y centro: Santa Fe, Rosario, Paraná y Córdoba. NEA: Corrientes y Posadas. Oeste: Mendoza, San Juan y La Rioja. NOA: Jujuy y Catamarca.

nicas dependen del lecho vascular afectado: en las coronarias se manifiesta por la aparición de síndrome coronario agudo, infarto agudo de miocardio (IAM) o muerte súbita; en el cerebro cursa clínicamente como un accidente cerebrovascular agudo (ACV) o como un accidente isquémico transitorio (AIT), y los episodios repetidos pueden desembocar en una demencia multiinfarto; y en las arterias periféricas, la expresión clínica es la claudicación intermitente o la isquemia aguda de los miembros inferiores ${ }^{4,5}$.

La aterosclerosis es un proceso crónico que se inicia en etapas muy tempranas de la vida. Algunos estudios sugieren que este proceso podría iniciarse antes del nacimiento, o en los primeros años de vida, y acentuarse con el correr del tiempo $0^{6,7}$.

Las causas exactas y los factores de riesgo (FR) de la aterosclerosis no son del todo conocidos; sin embargo, ciertas condiciones o hábitos pueden aumentar la posibilidad de desarrollarla. Entre ellos destacan: hipertensión arterial, tabaquismo, diabetes mellitus, obesidad, la edad y algunos componentes del estilo de vida contemporáneo, especialmente las actividades diarias con escasa demanda energética. Además, cambios en patrones alimentarios como consumo de productos manufacturados, ricos en grasas saturadas y azúcares simples, con escaso consumo de frutas y verduras explican al menos parcialmente la globalización de esta enfermedad ${ }^{8-10}$.

El conocimiento y detección de los FR desempeña un importante papel para la valoración del riesgo cardiovascular, pieza clave para las estrategias de intervención sobre dichas enfermedades ${ }^{4}$. La presencia de varios FR en un mismo individuo multiplica su riesgo de forma importante, teniendo un papel indiscutible en el cálculo de las puntuaciones de riesgo global y, por tanto, un gran peso en la prevención primaria y secundaria. Los hábitos y estilos de vida determinan la incidencia de muchos de esos factores, por lo cual su distribución se relaciona con las condiciones sociales y culturales específicas de cada población ${ }^{11}$, y deben ser considerados como las causas más importantes y modificables de la mayoría de las muertes por enfermedades cardíacas y ACV ${ }^{12}$.

Si bien los médicos en general tenemos la obligación de realizar promoción de la salud entre nuestros pacientes, los cardiólogos desempeñamos un rol preponderante, tanto por la mayor proximidad de la especialidad con estas patologías, como así también debido al papel
Tabla 1. Características basales de los encuestados

\begin{tabular}{lc} 
Características basales) & $\mathrm{n}=523$ \\
\hline Edad (años) [mediana (RIC)] & $31(28-38)$ \\
Sexo masculino [n (\%)] & $330(63,1 \%)$ \\
Cargo [n (\%)] & \\
$\quad$ Residente & $276(52,8 \%)$ \\
$\quad$ Jefe de Residentes & $20(3,8 \%)$ \\
Instructor de Residentes & $10(1,9 \%)$ \\
Fellow & $20(3,8 \%)$ \\
Especialista & $197(37,7 \%)$ \\
Año de residencia [n (\%)] & \\
$\quad$ Primero & $39(14,2 \%)$ \\
Segundo & $92(33,3 \%)$ \\
Tercero & $74(26,8 \%)$ \\
Cuarto & $71(25,7 \%)$
\end{tabular}

que ocupan nuestras aseveraciones en el imaginario colectivo. Pese a esto, varios registros han reportado que una proporción importante de médicos no ponen en práctica las recomendaciones que habitualmente realizan a sus pacientes.

Debido a esto nos propusimos describir los factores de riesgo cardiovascular (FRCV) presentes en residentes de Cardiología y cardiólogos de planta pertenecientes a residencias de Cardiología de todo el país, y relevar el grado de adherencia de los participantes del estudio a hábitos alimentarios saludables.

\section{MÉTODOS}

Estudio de corte transversal prospectivo, observacional y multicéntrico. Se incluyeron médicos cardiólogos en formación o especialistas, pertenecientes a residencias de Cardiología de todo el país, durante los meses de agosto-noviembre de 2017.

\section{POBLACIÓN}

Criterios de inclusión: médicos cardiólogos en formación o especialistas, pertenecientes a residencias de Cardiología de todo el país, independientemente de que se encuentren asociadas o no a CONAREC. Se definió como cardiólogos en formación a: residentes, concurrentes, becarios, jefes de residentes e instructores de residentes de servicios de Cardiología. Se consideró como médicos especialistas a todos aquellos cardiólogos que se desempeñen en una institución con residencia, o asociados a una institución con residencia de Cardiología. Asimismo se incluyó en este subgrupo a aquellos cardiólogos que se encontraban realizando un fellowship (especialización) o una residencia posbásica en Cardiología (es decir, que hayan concluido ya su residencia de Cardiología en cualquier institución del país o del exterior), aclarándose su condición.

No se realizó distinción entre fellowship y residencia posbásica.

Criterios de exclusión: médicos que se encontraban realizando o desempeñando una especialidad diferente de Cardiología, aun en el caso de que hubieran completado una residencia de Cardiología previamente. Asimismo se excluirán a aquellos profesionales que se encuentren jubilados de sus tareas.

\section{RECOLECCIÓN DE DATOS Y DEFINICIÓN DE VARIABLES}

La recolección de datos se llevó a cabo mediante entrevista personal o realización del cuestionario de forma autoadministrada 
Tabla 2. Perfil cardiovascular. IMC: índice de masa corporal.

\begin{tabular}{lc|}
\hline Factores de riesgo cardiovascular & $n=523$ \\
\hline Hipertensión arterial $(n ; \%)$ & $26(5 \%)$ \\
\hline Diabetes mellitus $(n ; \%)$ & $4(0.8 \%)$ \\
\hline Dislipemia $(n ; \%)$ & $75(14.3 \%)$ \\
\hline Ex tabaquismo $(n ; \%)$ & $66(12.6 \%)$ \\
\hline Tabaquismo $(n ; \%)$ & $88(16.8 \%)$ \\
\hline Categoría de peso según IMC $(n ; \%)$ & \\
$\quad$ Sobrepeso & $186(35.6 \%)$ \\
$\quad$ Obesidad & $59(11.3 \%)$ \\
Sedentarismo (n; \%) & $192(36.7 \%)$ \\
\hline Enfermedad cardiovascular $(n ; \%)$ & \\
Enfermedad coronaria & $5(1 \%)$ \\
Enfermedad cerebrovascular & $2(0.4 \%)$ \\
Enfermedad vascular periférica & $0(0 \%)$
\end{tabular}

(Apéndice I, Encuesta). La encuesta fue cerrada y prefijada, voluntaria y anónima. Se empleó para esto un muestreo no probabilístico por conveniencia.

La carga de datos se realizó on-line a través de una plataforma Web dedicada para tal fin -LimeSurvey-a través de un formulario electrónico (electronic case report form [eCRF]) diseñado especialmente con un acceso exclusivo a través de una clave individual para cada centro formador.

Las definiciones operativas de las variables relevadas en el presente estudio se definieron con anterioridad, y se brindaron pautas detalladas para la realización de las mediciones antropométricas

(Apéndice II, Definiciones operativas; y Apéndice III, Mediciones antropométricas).

\section{ANÁLISIS ESTADÍSTICO}

Las variables continuas fueron expresadas con media y desvío estándar o mediana y rango intercuartílico (RIC), dependiendo de su distribución. La normalidad de las mismas fue evaluada mediante herramientas gráficas (histogramas, gráficos de distribución normal, etc.) y el test de Shapiro-Wilk. Las variables categóricas se expresaron como números y porcentajes. Para las comparaciones entre grupos de las variables continuas con distribución normal se utilizó el test de Student. Cuando la distribución fue no gaussiana se aplicó el test de suma de rangos de Wilcoxon. Las comparaciones entre proporciones se efectuó mediante el test de Chi cuadrado o el test exacto de Fisher dependiendo de la frecuencia de valores esperados.

En todos los casos se asumió un error alfa del 5\% para establecer la significación estadística.

\section{CONSIDERACIONES ÉTICAS}

Debido a que los sujetos de estudio son pares o superiores de aquellos que desarrollan el presente estudio, y entendiendo que el anonimato de los participantes es un factor crucial para la veracidad de las respuestas proporcionadas, no se solicitará consentimiento informado por escrito. En cambio se considerará que, todos aquellos individuos que acepten participar de la encuesta, siendo ésta expresamente voluntaria, y teniendo libre acceso al presente protocolo, habrán prestado su conformidad para la misma.

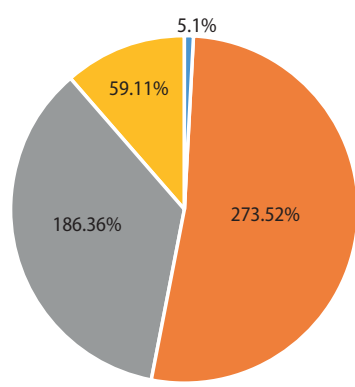

Bajo peso $\square$ Normopeso $\square$ Sobrepeso $\square$ Obesidad

Figura 2. Distribución de peso según índice de masa corporal (IMC). Clasificación: Bajo peso: IMC <18 kg/m². Normopeso: IMC $18-25 \mathrm{~kg} / \mathrm{m}^{2}$. Sobrepeso: IMC 25,1-29,9 kg/m². Obesidad: IMC $>30 \mathrm{~kg} / \mathrm{m}^{2}$

\section{RESULTADOS}

Se encuestaron 523 profesionales pertenecientes a 44 centros de todo el país. El 63,1\% de los participantes eran hombres, y la mediana de edad fue 31 años (RIC: 28-38 años) (Tabla 1). La distribución por regiones fue: Ciudad Autónoma de Buenos Aires 46,1\%, provincia de Buenos Aires 23,7\%, Litoral y Centro 13,2\%, Noreste Argentino 1\%, Oeste 12,4\% y Noroeste Argentino 3,6\% (Figura 1). El 52,8\% eran residentes, 5,9\% jefes o instructores de residentes y $41,3 \%$ especialistas o fellow.

Los principales FRCV detectados fueron: tabaquismo en el 16,8\% de los encuestados, dislipemia 14,3\%, hipertensión arterial 5\%, y diabetes mellitus tipo \| 0,8\% (Tabla 2). Así mismo eran ex tabaquistas 12,6\%. La mediana de edad de comienzo del hábito fue 18 años (RIC: 16-20) en las mujeres y 18 años (RIC: 16-22) en hombres; $\mathrm{p}=0,52$. Así mismo, la mediana de consumo de cigarrillos diarios entre mujeres fue 5 (RIC: 2-10) mientras que en hombres fue 7 (RIC: 3-15), sin ser esta diferencia estadísticamente significativa, $p=0,42$. Respecto a los antecedentes de enfermedad cardiovascular, el 1\% tuvo enfermedad coronaria, no se reportó enfermedad vascular periférica, y el 0,4\% enfermedad cerebrovascular.

La mediana del índice de masa corporal fue $21.7 \mathrm{~kg} / \mathrm{m}^{2}$ (RIC: 20,3-24) para mujeres y $26,1 \mathrm{~kg} / \mathrm{m}^{2}$ (RIC: $24,2-28,1$ ) en hombres. De acuerdo con esto, 46,9\% de los encuestados cumplían criterio de sobrepeso u obesidad (Figura 2)

Respecto a la proporción de tabaquistas de acuerdo al cargo desempeñado, eran fumadores activos 17,75\% de los residentes, $25,81 \%$ de los jefes o instructores de residentes, y 14,35\% de los médicos especialistas o fellows de los servicios ( $p=0,23)$. La proporción de extabaquistas fue $8 \%, 9,7 \%$ y $19 \%$ respectivamente $(p=0,001)$. Por otra parte, la mediana de edad de los no tabaquistas fue 30 años (RIC: 28-37), la de los tabaquistas 31 años (RIC: 29-35) y la de los extabaquistas 37,5 años (RIC: $32-50) ; p=0.0001$.

El 63,3\% de los participantes manifestó hacer algún tipo de actividad física, siendo el $87,6 \%$ recreativo, y el porcentaje restante a nivel competitivo. El 74,5\% realiza 2-3 días a la semana actividad física, con una carga horaria promedio semanal de 3.7 horas.

Además el 33,8\% expresó que no se cuidaba respecto al agregado sal a los alimentos y el 35,6\% no comían frutas ni verduras diariamente. 
Cuando se indagó acerca del último control de salud, el 41,9\% manifestó que fue había sido más de un año atrás.

\section{DISCUSIÓN}

Los médicos y en particular los cardiólogos, poseen formación sobre la modificación de los FRCV, la implicancia de los mismos, y los riesgos de eventos cardiovasculares vinculados a los mismos. Sin embargo, en la práctica clínica frecuentemente se pierde la oportunidad de su prevención, existiendo una brecha entre el conocimiento de los FRCV por parte del médico y la efectividad de los resultados del consejo preventivo. Las diferentes actitudes de los médicos frente a los FRCV pueden hacer variar el impacto de intervenciones en los pacientes. La alta frecuencia de factores de riesgo cardiovascular encontrados en nuestro estudio demuestra la falta de aplicación del conocimiento médico en su propia salud. Es imperativo encontrar estrategias para modificar los factores de riesgo cardiovascular en el equipo de salud, para prevenir la aparición de ECV y disminuir el impacto de esta.

En la Tercera Encuesta Nacional de Factores de riesgo para enfermedades no transmisibles en la población argentina publicada en el año 2015, podemos evidenciar que la prevalencia de consumo de tabaco fue del $25,1 \%$, siendo mayor en el sexo masculino (29,9 vs. 20,9\%) y más evidente en el grupo etario de los 25 a los 34 años $(30,8 \%)^{13}$. En relación a nuestro estudio en donde la edad media (31 años) coincide con el grupo etario que más tabaco consume, la prevalencia de tabaquismo fue significativamente menor (16,8\%).

Con respecto a la dislipidemia la comparación entre ambos estudios se dificulta, ya que en la Tercera Encuesta Nacional toma como parámetros hombres mayores de 35 años y mujeres mayores de 45 años, con una prevalencia de dislipidemia de $77,5 \%$, mientras que en nuestro estudio encontramos una prevalencia de $14,3 \%$ pero en una población significativamente más joven. Lo mismo ocurre con la prevalencia de hipertensión arterial (34,1 vs. 5\%) y diabetes mellitus (9,8 vs. $0,8 \%)$, comparaciones no valorables con la diferencia de edad en la población en comparación.

En el estudio RENATA la prevalencia de HTA en la población general fue de 33,5\%, pero en menores de 35 años fue de $11,1 \%{ }^{14}$, siendo menor la encontrada entre los participantes de nuestro trabajo (5\%). De forma similar, en el estudio RENATA 2 la prevalencia de HTA en menores de 35 años fue 12,2\%, mayor que la reportada Estudio RENATA 1, realizado 5 años antes ${ }^{15}$. Comparando ambos estudios con nuestra encuesta encontramos una prevalencia de HTA mucho menor entre médicos cardiólogos y residentes.

La encuesta publicada por Intramed sobre detección del riesgo cardiovascular en médicos argentinos realizada entre los año 2005 y 2006 mostró una tasa de tabaquismo en profesionales de la salud de 23,22\% ${ }^{16}$, lo cual resultó menor que la comunicada en la población general para esos años, al igual que lo que ocurre en nuestro trabajo. Sin embargo, por tratarse de poblaciones diferentes no es posible asegurar que exista una tendencia real hacia un menor consumo de tabaco en los últimos años.
Un dato llamativo de la encuesta de Intramed fue que el 54,9\% de los profesionales encuestados refirió tener sobrepeso, siendo esto mayor que en la población general. En nuestro trabajo la prevalencia fue algo menor, pero con una media de edad más joven. Ambas observaciones resultan alarmantes, y deberían implementarse estrategias para revertir esto.

Más preocupante aún que la proporción de factores de riesgo encontrados es la alta tasa de estilos de vida no saludable en nuestra muestra. Globalmente casi 1 de cada 3 participantes de la encuesta no realizaba actividad física, no procuraban reducir el consumo de sodio diario, ni comían frutas y verduras diariamente. Además la mitad de los participantes negaban haber realizado un control médico de cualquier tipo en el último año. Tratándose de una población eminentemente joven, es dable suponer que de persistir estas tendencias, exista un progresivo aumento de los FRCV en los próximos años.

Nuestro estudio posee algunas limitaciones que merecen ser resaltadas: en primer término, al tener un muestreo no probabilístico resulta imposible establecer con precisión la prevalencia de los FRCV en la población estudiada. Ligado a esto merece destacarse que, debido a un mayor número de centros formadores en CABA y Buenos Aires, existe una desproporción entre los participantes de estas regiones, comparados con las restantes. En segundo lugar, mucha información recabada fue referida por los participantes, sin corroborar las respuestas. Algo similar ocurre con las mediciones antropométricas, ya que se emplearon para ellas diferentes instrumentos (según la disponibilidad de cada servicio), sin haber corroborado en todos los casos la adecuada calibración de los mismos.

Sin embargo, siendo todos los participantes profesionales de la salud, es dable suponer que los posibles errores vinculados a esto sean menores. Además, el tamaño muestral grande del estudio compensa parcialmente algunas de las debilidades antes enumeradas.

\section{CONCLUSIÓN}

Encontramos una elevada tasa de factores de riesgo cardiovascular entre los participantes, considerando la edad y profesión de los mismos. Los principales factores detectados fueron el sobrepeso y obesidad y el sedentarismo, seguidos de la adicción al tabaco y la dislipidemia. Si bien la tasa de otros factores de riesgo como hipertensión arterial y diabetes fue baja, debe tenerse presente que los participantes fueron en su mayoría jóvenes.

Respecto al tabaquismo, no encontramos diferencias sustanciales entre cardiólogos en formación y especialistas.

Creemos que estos hallazgos deben ser analizados con especial atención, habida cuenta que ciertas prácticas se ven condicionadas por las condiciones laborales y la remuneración económica percibida, como resulta el caso de la alimentación o la práctica de actividad física.

Consideramos esencial implementar estrategias para favorecer estilos de vida saludables entre profesionales de la salud de nuestro país. Sería deseable repetir esta evaluación en un tiempo prudencial, para valorar las tendencias temporales en los factores de riesgo y estilos de vida entre los residentes de Cardiología y cardiólogos del país. 


\section{BIBLIOGRAFÍA}

1. Navarro-López F. Bases genéticas de la enfermedad coronaria. Rev Esp Cardiol 2002;55(4):413-31.

2. Priori SG, Blomström-Lundqvist C, Mazzanti A, Blom N, Borggrefe M, Camm J, et al. 2015 ESC Guidelines for the management of patients with ventricular arrhythmias and the prevention of sudden cardiac death: The Task Force for the Management of Patients with Ventricular Arrhythmias and the Prevention of Sudden Cardiac Death of the European Society of Cardiology (ESC). Endorsed by: Association for European Paediatric and Congenital Cardiology (AEPC). Eur Heart J 2015;36(41):2793-867.

3. Touboul PJ, Hennerici MG, Meairs S, Adams H, Amarenco P, Bornstein N, et al. Mannheim carotid intima-media thickness and plaque consensus (2004-20062011). An update on behalf of the advisory board of the 3rd, 4th and 5th watching the risk symposia, at the 13th, 15th and 20th European Stroke Conferences, Mannheim, Germany, 2004, Brussels, Belgium, 2006, and Hamburg, Germany, 2011. Cerebrovasc Dis 2012;34(4):290-6

4. Lahoz C, Mostaza J. La aterosclerosis como enfermedad sistémica. Rev Esp Cardiol 2007;60(2):184-95.

5. Aguiar-Souto P, Cabanas-Grandío P, González-Juanatey JR. La enfermedad polivascular aterotrombótica: un enemigo común y distintos frentes. Rev Esp Cardiol 2009;9(D):4-10.

6. Palinski W, Napoli C. Pathophysiological events during pregnancy influence the development of atherosclerosis in humans. Trends Cardiovasc Med 1999;9(7):205-14.

7. Napoli C, D'Armiento FP, Corso G, Ambrosio G, Palumbo G, Zuliani P, et al. Occurrence of the same peroxidative compounds in low density lipoprotein and in atherosclerotic lesions from a homozygous familial hypercholesterolemic patient: a case report. Int J Cardiol 1997;62(1):77-85.
8. Weber C, Noels H. Atherosclerosis: current pathogenesis and therapeutic options. Nat Med 2011:17(11):1410-22

9. Owen DR, Lindsay AC, Choudhury RP, Fayad ZA. Imaging of atherosclerosis. Annu Rev Med 2011;62:25-40.

10. Ross R. The pathogenesis of atherosclerosis: A perspective for the 1990s. Nature 1993;362(6423):801-9.

11. Lastiri H, Flichtentrei D. Prevención cardiovascular en el siglo XXI: construyendo nuevos escenarios para la acción Sociedad de Cardiología del Oeste Bonaerense. Federación Argentina de Cardiología.

12. Pearson TA, Bazzarre TL, Daniels SR, Fair JM, Fortmann SP, Franklin BA, et al. American Heart Association Guide for Improving Cardiovascular Health at the Community Level. Circulation 2002;107(4):645-51.

13. Ministerio de Salud de la Nación. Tercera Encuesta Nacional de Factores de Riesgo para enfermedades no transmisibles. Argentina 2013.

14. Marin MJ, Fábregues G, Rodríguez PD, Díaz M, Páez O, Alfie J, et al. Registro Nacional de Hipertensión Arterial. Conocimiento, tratamiento y control de la hipertensión arterial. Estudio RENATA. Rev Argent Cardiol 2012;80(2):121-9.

15. Delucchi AM, Majul CR, Vicario A, Cerezo GH, Fábregues G. Registro Nacional de Hipertensión Arterial. Características epidemiológicas de la hipertensión arterial en Argentina. Estudio RENATA 2. Rev Argent Cardiol 2017;85(4):354-60.

16. Zulma O, Luna D, Braga F, Prats M, Flichtentrei D. Estudio Doctor: Detección del riesgo cardiovascular en médicos argentinos. Intramed Investigaciones 2009. Disponible en https://www.intramed.net/userfiles/2009/file/estudio_ doctor.pdf. 